\title{
Dynamical and anisotropic properties of spin-VCSELs
}

M. Drong, T. Fördös, H. Jaffrès, K. Postava, J. Peřina, et al.

M. Drong, T. Fördös, H. Jaffrès, K. Postava, J. Peřina Jr., H.-J Drouhin, J. Pištora, "Dynamical and anisotropic properties of spin-VCSELs," Proc. SPIE 10926, Quantum Sensing and Nano Electronics and Photonics XVI, 1092614 (1 February 2019); doi: 10.1117/12.2515288

SPIE. Event: SPIE OPTO, 2019, San Francisco, California, United States 


\title{
Dynamical and anisotropic properties of spin-VCSELs
}

\author{
(Invited)
}

\author{
M. Dronga,b, T. Fördös ${ }^{a, b, c}$, H. Jaffrès ${ }^{\mathrm{d}}$, K. Postava ${ }^{\mathrm{a}, \mathrm{b}}$, J. Peřina Jr. ${ }^{\mathrm{e}}$, H.-J. Drouhin ${ }^{\mathrm{c}}$, and \\ J. Pištora ${ }^{a, b}$

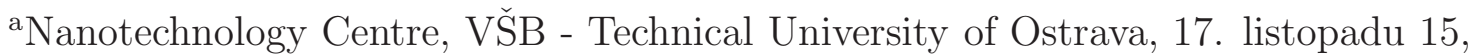 \\ 70833 Ostrava-Poruba, Czech Republic \\ bIT4Innovation, VŠB - Technical University of Ostrava, 17. listopadu 15, \\ 70833 Ostrava-Poruba, Czech Republic \\ ${ }^{\mathrm{c}}$ Laboratoire des Solides Irradiés, École Polytechnique, CNRS, CEA-DSM-IRAMIS, \\ Université Paris-Saclay, 91128 Palaiseau Cedex, France \\ ${ }^{\mathrm{d} U n i t e ́ ~ M i x t e ~ d e ~ P h y s i q u e ~ C N R S / T h a l e s ~ a n d ~ U n i v e r s i t e ́ ~ P a r i s-S a c l a y, ~} 1$ Avenue A. Fresnel, \\ 91767 Palaiseau Cedex, France \\ e Joint Laboratory of Optics of Palacky University and Institute of Physics of Academy of \\ Sciences of the Czech Republic, Palacky University, 17.listopadu 50A, 77207 Olomouc, \\ Czech Republic
}

\begin{abstract}
Spin-polarized lasers such as spin-polarized vertical-cavity surface-emitting laser (spin-VCSELs) are prospective devices in which the radiative recombination of spin-polarized carriers results in an emission of circularly-polarized photons. Nevertheless, additional linear in-plane anisotropies in the cavity generally lead in preferential linearlypolarized laser emission and to possible coupling between modes. Optimization of room-temperature spinVCSELs thus relies on a proper modeling method and on a good understanding of these anisotropies that may reveal (i) a local linear birefringence due to strain fields at the surface or (ii) a birefringence in quantum wells (QWs) due to phase-amplitude coupling originating from the reduction of the biaxial $D_{2 d}$ to the $C_{2 v}$ symmetry group at the III-V ternary semiconductor interfaces. We present a novel method for the modeling of steady-state and dynamical properties of generally anisotropic multilayer semiconductor lasers containing multiple QWs active region. In order to solve the dynamical properties of spin-VCSELs, we combine here optical Bloch equations for a 4-level system with the scattering-matrix formalism, which treats VCSELs as a multilayer structure containing classical active dipole layers [T. Fördös et al., Phys. Rev. A 96, 043828 (2017)]. The method is then demonstrated on real semiconductor laser structures with InGaAs/GaAsP quantum wells. It is used for calculation of the laser resonance condition, the polarization properties of eigenmodes, the electromagnetic-field distribution inside the laser cavity, and time-dependent properties of the emitted light.
\end{abstract}

Keywords: spin-VCSEL, dynamical properties, scattering matrix formalism, Bloch equations

\section{INTRODUCTION}

Due to advances in nanostructure preparation techniques in the recent years, it is now possible to prepare systems in which the quantum nature of matter and fields plays a significant role. It is for example the modification of spontaneous emission using nanostructured photonic crystals, where photonic density of states is basically engineered. Considering laser technologies, it is promising technology of vertical (external)-cavity surface-emitting lasers [V(E)CSELs]. ${ }^{1}$ Active zones of such devices consist of semiconductor quantum wells (QWs) or quantum dots (QDs) with quantized energy spectrum due to reduced dimensions at least in one spatial direction. Here, we limit ourselves to semiconductor QWs. A process of stimulated emission requires the recombination of conduction and valence band electronic states governed by optical selection rules. Laser cavity of monolithic VCSEL

Further author information: Mariusz Drong, e-mail: mariusz.drong@vsb.cz 
contains top and bottom distributed Bragg reflectors (DBRs) of very high reflectivity that originates from the existence of photonic band gap in DBR's spectra. ${ }^{2}$ On the other hand, VECSELs consist of a single DBR at the bottom of the structure and an external mirror placed in significant distance from a laser chip. From a material point of view, $\mathrm{V}(\mathrm{E}) \mathrm{CSEL}$ technologies are based on binary and ternary III-V semiconductor compounds.

The architecture and properties of V(E)CSEL structures are suitable for industrial realization of so-called spin-polarized lasers (or simply spin-lasers). ${ }^{3,4}$ Energy states in semiconductor QWs are degenerated in a spin degree of freedom. However, this degeneracy can be lifted using spin-injection into QWs, which can be realized using spin-polarized electrical current and/or appropriately polarized optical pump. ${ }^{5,6}$ According to Fig. 1 (right), which shows 4-level active media of spin-laser (considering only conduction electrons and heavy holes), it is possible, in principle, to control the polarization of emitted field by lifting degeneracy of the electron spin projections using the spin-injection. ${ }^{7} \sigma_{ \pm}$stand for the transitions of circularly-polarized photons.

Spin-lasers promise improved performance compared to their conventional counterparts. ${ }^{3,4}$ Namely, one can expect the threshold pumping rate reduction, which is already routinely demonstrated in laboratories around the world. However, desired and theoretically predicted $50 \%$ threshold reduction is difficult to obtain due to very short spin relaxation lifetime. In practice, the polarization control is affected by the presence of optical anisotropies in the laser cavity, which is of the main interest of this work. Strong anisotropies, such as residual linear birefringence and linear gain dichroism at semiconductor/air interface and in QWs, respectively, make linear polarization preferable. ${ }^{8-11}$ Actually, there are successful approaches to overcome this phenomena, e.g. placing birefringent optical element inside laser cavity to compensate residual birefringence of laser. Moreover, the oscillation of elliptical and also circularly-polarized laser eigenmodes was observed. ${ }^{6,11,12}$ Such approaches are not practical for integration of spin-laser with components of integrated optics. Another consequence of anisotropies is significant coupling between laser eigenmodes which results, for example, in time-domain modulation of emitted degree of circular polarization. ${ }^{13}$ This strongly influence stability of laser eigenmodes. ${ }^{6,8,10}$ Enhanced modulation dynamics of spin-lasers is also intensively studied. ${ }^{14}$ Recently, it was demonstrated that several times higher bit rate can be obtained with spin-lasers. ${ }^{15}$

Different modeling tools are currently available to predict steady-state and dynamical properties of spinlasers. Qualitatively correct analysis of spin-laser eigenmodes in the presence of optical anisotropies was recently performed using $2 \times 2$ Jones matrices revealing for example possible compensation of linear anisotropies using spin-injection. ${ }^{16}$ However, such approaches operate with effective quantities distributed through entire laser structure, which is not practical for design of future spin-lasers. Sophisticated layer-by-layer treatment of spinlasers [spin-V(E)CSELs especially] based on scattering matrices was currently proposed by Fördös et al. ${ }^{17,18}$ Such approach allows to extract and study laser eigenmodes of arbitrary spin-V(E)CSEL with localized linear birefringence and linear dichroism and consequently allows possible design of future spin-lasers. ${ }^{19}$ In the present paper, we extend this work. Dynamical properties of spin-lasers, on the other hand, are studied in the framework of the spin-flip model. Excellent agreement with experiment was obtained using spin-flip model in the recent years. ${ }^{14}$ However, also spin-flip model uses effective quantities describing linear optical anisotropies. Here, we include optical anisotropies as localized phenomena also in the context of laser dynamics.

In the Sec. 2 we introduce the description of anisotropic spin-lasers in the framework of the generalized Maxwell-Bloch equations. We present alternative derivation of gain tensor and amplification matrix ${ }^{18}$ used in the matrix formalism of Fördös et al. for extraction of laser eigenmodes. Sec. 2 is dedicated also for description of the method for the calculation of electromagnetic field distributions in anisotropic spin-V(E)CSELs. Next, we formulate the rate-equation model derived directly from the generalized Maxwell-Bloch equations, which describes oscillation of two modes in a spin-polarized V(E)CSEL with localized optical anisotropies. In the Sec. 3, we apply our modeling tools to a real spin-VECSEL with linear gain anisotropy in QWs and linear birefringence at the semiconductor/air interface. We calculate electromagnetic field distribution inside the laser structure considering the optical anisotropies and spin-injection. Finally, the rate-equation model respecting localized nature of the anisotropies is used to determine dynamical properties of the spin-VECSEL involving the linear gain dichroism. 

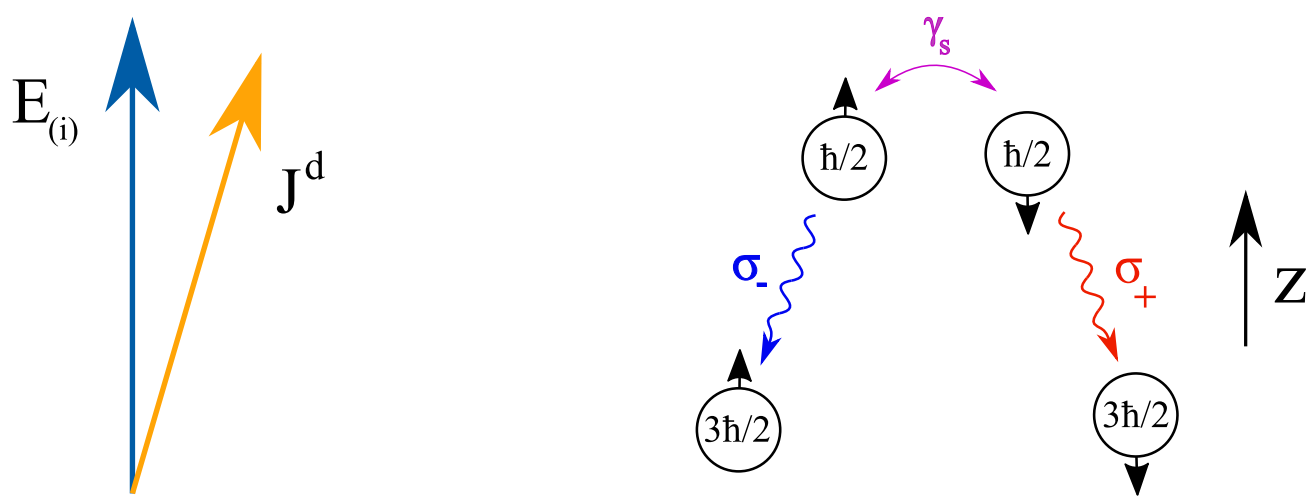

Figure 1. (left) The polarization of laser eigenmode $\mathbf{E}_{(i)}$ and polarization of optical transition inside active media described by the Jones source vector $\mathbf{J}^{d}$ do not have to be necessarily collinear. Non-collinearity may be caused by optical anisotropies inside laser cavity and/or by anisotropic nature of active media. In a case of semiconductor QW with linear gain dichroism, it is related to the non-orthogonality of Jones source vectors $\mathbf{A}_{ \pm}^{d}$ related to $N_{ \pm}$populations. ${ }^{18}$ (right) 4-level electronic system of semiconductor QW with two possible optical transitions. ${ }^{7}$

\section{THEORETICAL BASIS}

\subsection{Active Media Dynamics, Generalized Maxwell-Bloch Equations}

Dynamics of interaction between active media and electromagnetic field in the dipole approximation can be described by the Maxwell-Bloch equations, sometimes called the optical Bloch equations. ${ }^{20}$ Considering generally multimode oscillation, they couple together the electric field intensity $\mathbf{E}_{(i)}$, the electric dipole moment density $\mathbf{P}_{(i)}$ of $i$-th mode, and the population inversion variables $N_{q}$, where index $q$ defines carrier channel or type of the optical transition. In the case of spin-polarized lasers $q \in\{+,-\}$, referring to different spin projections of conduction band electrons. One of the advantages of optical Bloch equations related to modeling of an anisotropic laser structures such as birefringent spin-V(E)CSELs is respecting of vectorial nature of the electric field intensity and the macroscopic polarization P. As shown in Fig. 1 (left), laser eigenmodes are generally not collinear with the Jones vector characterizing active media eigenpolarization. It is due to optical anisotropies present in a laser cavity and due to anisotropic nature of laser active media. In fact, it means that in a case of such non-collinearity only fraction of incoming electromagnetic field is amplified during stimulated emission.

Mathematically, it is realized by projecting the electric field intensity vector of laser eigenmode on directions of the active media Jones source vectors. ${ }^{21}$ Interaction of multiple modes of electromagnetic field with the spin-polarized carrier densities $N_{ \pm}$in an anisotropic laser cavity is described by the generalized Maxwell-Bloch equations $^{18}$

$$
\begin{gathered}
\frac{\partial \mathbf{P}_{(i)}}{\partial t}=-(\Gamma+\mathrm{i} \delta) \mathbf{P}_{(i)}-\mathrm{i} \frac{\mu^{2}}{\hbar}\left[\left(\mathbf{E}_{(i)} \cdot \mathbf{A}_{+}^{\mathrm{d} *}\right) \mathbf{A}_{+}^{\mathrm{d}} \mathrm{N}_{+}+\left(\mathbf{E}_{(i)} \cdot \mathbf{A}_{-}^{\mathrm{d} *}\right) \mathbf{A}_{-}^{\mathrm{d}} \mathrm{N}_{-}\right], \\
\frac{\partial N_{ \pm}}{\partial t}=-\gamma\left(N_{ \pm}-N_{0 \pm}\right) \mp \gamma_{s}\left(N_{+}-N_{-}\right)-\frac{\mathrm{i}}{\hbar} \sum_{i}\left\{\left(\mathbf{E}_{(i)}^{*} \cdot \mathbf{A}_{ \pm}^{d}\right)\left(\mathbf{P}_{(i)} \cdot \mathbf{A}_{ \pm}^{d *}\right)-c . c .\right\} \\
\frac{\partial^{2}}{\partial t^{2}} \mathbf{P}_{(i)} \exp \left[\mathrm{i}\left(\omega \mathrm{t}-\mathrm{k}_{(i)} \mathrm{z}\right)\right]=\left[c^{2} \nabla^{2}-\varepsilon \frac{\partial^{2}}{\partial t^{2}}-\kappa \frac{\partial}{\partial t}\right] \mathbf{E}_{(i)} \exp \left[\mathrm{i}\left(\omega \mathrm{t}-\mathrm{k}_{(i)} \mathrm{z}\right)\right],
\end{gathered}
$$

where the first of them, Eq. (1), describes the time-dependece of macroscopic polarization $\mathbf{P}_{(i)}$ induced by interaction of the field mode $\mathbf{E}_{(i)}$ with both carrier channels. The macroscopic polarization decays with the rate $\Gamma$. Parameter $\delta$ represents the spectral detuning and $\mu$ is the off-diagonal matrix element of the electric dipole operator. Eq. (2) describes dynamics of the population inversions $N_{ \pm}$pumped with the rates $\gamma N_{0 \pm}$, where $\gamma$ stands for the population decay rate coefficient. Spin-mixing processes can be also described by the effective coefficient $\gamma_{s}$. Equation (3) connects active media to the rest of the laser structure by describing propagation of eigenmode of the frequency $\omega$ in entire laser cavity and its dynamics. Decay of laser eigenmodes is described 
by $\kappa$ which is defined as $1 / \tau_{p h}$, where $\tau_{p h}$ stands for the photon lifetime inside cavity. Optical properties of the cavity are described by the permittivity $\varepsilon$, which is generally different for each layer.

As shown in Fig. 1, non-orthogonality of the Jones source vectors $\mathbf{A}_{ \pm}^{d}$ is caused by the presence of linear gain anisotropy in semiconductor QW which is induced by reduced crystallographic symmetry at quantum well/barrier interfaces. ${ }^{22}$ In order to evaluate the exact form of Jones source vectors in an active media with linear gain dichroism, let us define the dipolar transition matrix elements $\Pi_{x^{\prime}}, \Pi_{y^{\prime}}$ along $x^{\prime}=[110]$ and $y^{\prime}=[1 \overline{1} 0]$ axes (see Fig. 4). We introduce parameter $1-\Delta$ which quantifies linear gain anisotropy. ${ }^{18}$ It follows that two orthogonal dipolar matrix elements $\Pi_{x^{\prime}}, \Pi_{y^{\prime}}$ are linked together as $\Pi_{y^{\prime}}=\Delta \Pi_{x^{\prime}}$. It means that the dipolar interaction has different strength along $x^{\prime}$ and $y^{\prime}$. In the reference frame defined by basis axes $x=[100]$ and $y=[010]$, the Jones source vectors characterizing optical transitions in $(+)$ and $(-)$ channels are ${ }^{18}$

$$
\mathbf{A}_{+}^{d}=\mathbf{A}_{-}^{d *}=\frac{1}{\sqrt{1+\Delta^{2}}}\left[\begin{array}{c}
\frac{1+\Delta}{2}-\mathrm{i} \frac{1-\Delta}{2} \\
-\mathrm{i} \frac{1+\Delta}{2}+\frac{1-\Delta}{2}
\end{array}\right] .
$$

Note that for $\Delta=1$, which corresponds, according to our convention, to vanishing linear gain anisotropy, the Jones source vectors describe orthogonal circularly-polarized transitions $\sigma_{+}$and $\sigma_{-}$, as shown in Fig. 1 . For $\Delta \neq 1$ vectors $\mathbf{A}_{ \pm}^{d}$ are not orthogonal to each other, resulting in a strong mode coupling between laser eigenmodes.

Note that the laser eigenmodes $\mathbf{E}_{(i)}$ are determined not only by properties of active media such as spin polarization $P_{s}$ or linear gain dichroism $\Delta$, but additionally by optical properties of entire laser cavity. ${ }^{18}$ In order to find such laser eigenmodes we will introduce the required matrix formalism in the next parts of this paper.

\subsection{Amplification Matrix}

We will adapt the description of light interaction with semiconductor QWs based on the generalized MaxwellBloch equations presented in Sec. 2.1 for a need of matrix method which will enable us for example to find laser eigenmodes. Using slowly varying envelope approximation and adiabatic elimination of the dipole moment density $\mathbf{P}_{(i)}$ valid for Class-A and Class-B lasers, ${ }^{20,21}$ we simplify Equation (3) into ${ }^{18}$

$$
\frac{d \mathbf{E}_{(i)}}{d t}=-\frac{1}{2} \kappa \mathbf{E}_{(i)}+\frac{\omega \mu^{2}}{2 \varepsilon(\Gamma+\mathrm{i} \delta) \hbar}\left[\left(\mathbf{E}_{(i)} \cdot \mathbf{A}_{+}^{d *}\right) \mathbf{A}_{+}^{d} N_{+}+\left(\mathbf{E}_{(i)} \cdot \mathbf{A}_{-}^{d *}\right) \mathbf{A}_{-}^{d} N_{-}\right]
$$

where the first term represents cavity losses and the second term represents interaction of $i$-th mode of the electromagnetic field $\mathbf{E}_{(i)}$ with carriers $N_{+}$and $N_{-}$. For simplicity, we use notation $g=\frac{\omega \mu^{2}}{2 \varepsilon(\Gamma+\mathrm{i} \delta) \hbar}$ for the interaction constant. ${ }^{18}$ Next, we consider the general electric field intensity vector $\mathbf{E}_{(i)} \rightarrow \mathbf{E}$. Using methods of tensor calculus, it can be shown that Equation (5) can be re-expressed as ${ }^{18,21}$

$$
\frac{d \mathbf{E}}{d t}=-\frac{1}{2} \kappa \mathbf{E}+g\left[\left(\mathbf{A}_{+}^{d} \otimes \mathbf{A}_{+}^{d *}\right) N_{+}+\left(\mathbf{A}_{-}^{d} \otimes \mathbf{A}_{-}^{d *}\right) N_{-}\right] \mathbf{E}
$$

where we have introduced the gain operator $\hat{\mathcal{T}}=\left[\left(\mathbf{A}_{+}^{d} \otimes \mathbf{A}_{+}^{d *}\right) N_{+}+\left(\mathbf{A}_{-}^{d} \otimes \mathbf{A}_{-}^{d *}\right) N_{-}\right]$. Note that we are searching for matrix description of monochromatic light passing through an anisotropic QW. For this reason, we go from time to space domain using $\frac{d \mathbf{E}}{d t}=\frac{d \mathbf{E}}{d z} \frac{d z}{d t}$, where $\frac{d z}{d t}=v=\frac{c}{n}$ is speed of considered electromagnetic wave inside QW of the refractive index $n$. Since a phenomenological description of cavity losses takes into account all of loss mechanisms of photons in a resonator, we are allowed to neglect the loss term during short interval in which photons pass through QW. We can write

$$
\frac{c}{n} \frac{d \mathbf{E}}{d z}=g \hat{\mathcal{T}} \mathbf{E}
$$

which can be solved by conventional methods under the assumption that population inversion is fixed to its threshold value. For a QW of the thickness $W$ we have

$$
\mathbf{E}_{\text {out }}=\exp \left[\frac{n W g}{c} \hat{\mathcal{T}}\right] \mathbf{E}_{\text {in }}
$$


where $\mathbf{E}_{\text {out }}$ and $\mathbf{E}_{\text {in }}$ are intensities of the electric field outgoing from and incoming to QW, respectively. Due to very small thickness of $\mathrm{QW}$ we use a linear approximation valid also for arbitrary operator $\hat{A}$ : $\exp [\hat{A}] \cong \hat{I}+\hat{A}$, if $\langle\hat{A}\rangle<<1$, where $\hat{I}$ stands for the unity operator. We obtain the operator expression connecting the electric field components outgoing from and incoming to single QW

$$
\mathbf{E}_{\text {out }}=\left[\hat{I}+\frac{n W g}{c} \hat{\mathcal{T}}\right] \mathbf{E}_{\text {in }}
$$

where it is useful to re-define the gain tensor as $\mathcal{T}=\hat{\mathcal{T}} / N:^{18}$

$$
\mathcal{T}=\frac{N_{+}}{N_{+}+N_{-}}\left(\mathbf{A}_{+}^{d} \otimes \mathbf{A}_{+}^{d *}\right)+\frac{N_{-}}{N_{+}+N_{-}}\left(\mathbf{A}_{-}^{d} \otimes \mathbf{A}_{-}^{d *}\right)
$$

and introduce the gain coefficient $g_{0}{ }^{18}$

$$
g_{0}=\frac{n W g N}{c}=\frac{n W \omega \mu^{2} N}{2 \varepsilon c(\Gamma+\mathrm{i} \delta) \hbar}
$$

where $N=N_{+}+N_{-}$. We may represent unity operator using the $2 \times 2$ unity matrix $\mathbf{I}$. Additionally, since we are considering semiconductor active media, we should use the Henry's coefficient $\alpha$ to describe changes in the real part of the refractive index due to pumping of the carriers into QW. ${ }^{23}$ Finally, using Eqs. (10) and (11), Eq. (9) becomes ${ }^{18}$

$$
\mathbf{E}_{\text {out }}=\left[\mathbf{I}+g_{0}(1-\mathrm{i} \alpha) \mathcal{T}\right] \mathbf{E}_{\text {in }}=\mathbf{T}_{\mu \mu} \mathbf{E}_{\text {in }},
$$

where $\mathbf{T}_{\mu \mu}$ are the $2 \times 2$ components of amplification matrix $\mathbf{T}$ and where index $\mu \in\{u, d\}$ defines up- and downtravelling amplitudes. In the framework of the scattering matrix formalism, we can describe light amplification in a single semiconductor $\mathrm{QW}$ as ${ }^{18}$

$$
\left[\begin{array}{l}
\mathbf{A}_{u} \\
\mathbf{A}_{d}
\end{array}\right]_{\text {out }}=\left[\begin{array}{cc}
\mathbf{T}_{u u} & \mathbf{0} \\
\mathbf{0} & \mathbf{T}_{d d}
\end{array}\right]\left[\begin{array}{l}
\mathbf{A}_{u} \\
\mathbf{A}_{d}
\end{array}\right]_{i n}
$$

The amplification matrix $\mathbf{T}$ relates amplitudes outgoing from and incoming to QW.

\subsection{Propagation in Optical Cavity, Extraction of Laser Eigenmodes}

In order to model emission from a $\mathrm{V}(\mathrm{E}) \mathrm{CSEL}$, it is necessary to describe also propagation in the rest of the laser cavity. We devide the structure into three blocks: ${ }^{17,18}$ the upper Bragg mirror (or external mirror in the case of 1/2-VCSEL), the active zone and the lower Bragg mirror as shown in Fig. 2. Let us begin with the description of light interaction in the semiconductor multiple QW (MQW) active region. Respecting the notation in Fig. 2,
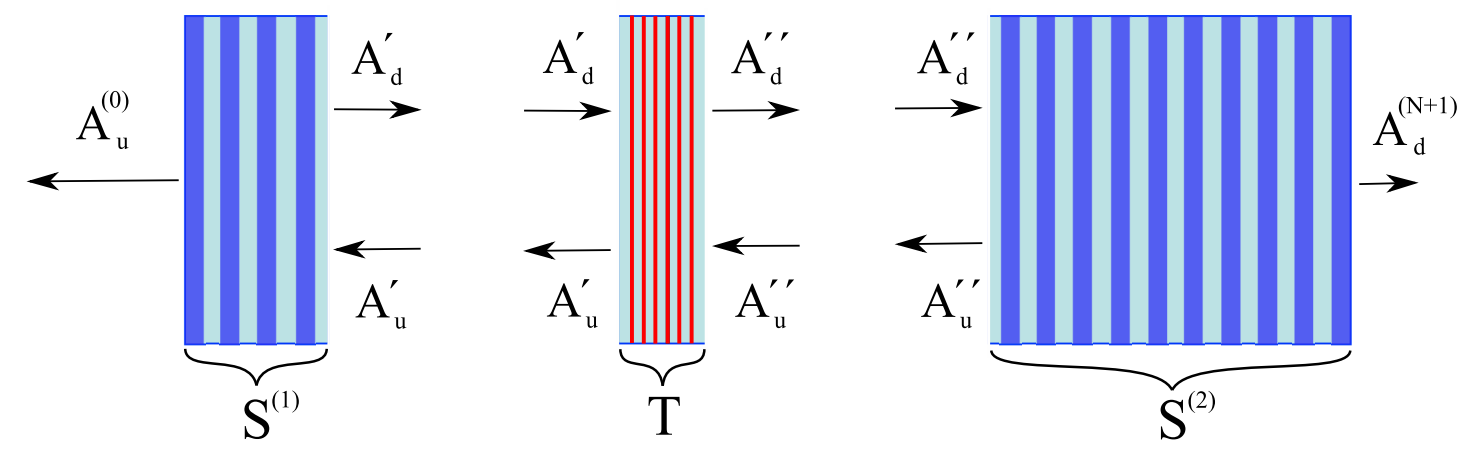

Figure 2. The approach used for solving the problem of light propagation and amplification in a complex anisotropic $\mathrm{V}(\mathrm{E}) \mathrm{CSEL}$ or spin-V(E)CSEL. Laser structure is devided into three parts which are described separately. 
we relate amplitudes interacting with active zone using the amplification matrix $\mathbf{T}$ derived in Sec. 2.2 in the following way ${ }^{18}$

$$
\left[\begin{array}{c}
\mathbf{A}_{u}^{\prime} \\
\mathbf{A}_{d}^{\prime \prime}
\end{array}\right]=\left[\begin{array}{ll}
\mathbf{T}_{u u} & \mathbf{T}_{u d} \\
\mathbf{T}_{d u} & \mathbf{T}_{d d}
\end{array}\right]\left[\begin{array}{l}
\mathbf{A}_{u}^{\prime \prime} \\
\mathbf{A}_{d}^{\prime}
\end{array}\right],
$$

where also off-diagonal submatrices $\mathbf{T}_{u d}$ and $\mathbf{T}_{d u}$ appear due to reflection events in MQW. ${ }^{18}$ In the framework of scattering matrix formalism the off-diagonal elements stand for reflectivity matrices. We use scattering matrices $\mathbf{S}^{(1)}$ and $\mathbf{S}^{(2)}$ to describe light propagation in upper and lower mirrors respectively. Definition of S-matrices is presented in Appendix A. According to Fig. 2 we can write ${ }^{18}$

$$
\begin{gathered}
{\left[\begin{array}{c}
\mathbf{A}_{u}^{(0)} \\
\mathbf{A}_{d}^{\prime}
\end{array}\right]=\left[\begin{array}{ll}
\mathbf{S}_{u u}^{(1)} & \mathbf{S}_{u d}^{(1)} \\
\mathbf{S}_{d u}^{(1)} & \mathbf{S}_{d d}^{(1)}
\end{array}\right]\left[\begin{array}{c}
\mathbf{A}_{u}^{\prime} \\
\mathbf{0}
\end{array}\right],} \\
{\left[\begin{array}{c}
\mathbf{A}_{u}^{\prime \prime} \\
\mathbf{A}_{d}^{(N+1)}
\end{array}\right]=\left[\begin{array}{ll}
\mathbf{S}_{u u}^{(2)} & \mathbf{S}_{u d}^{(2)} \\
\mathbf{S}_{d u}^{(2)} & \mathbf{S}_{d d}^{(2)}
\end{array}\right]\left[\begin{array}{c}
\mathbf{0} \\
\mathbf{A}_{d}^{\prime \prime}
\end{array}\right],}
\end{gathered}
$$

where we use the fact that electromagnetic field radiates only out of the structure. In the next step, we solve Eqs. (14), (15) and (16) to obtain emitted amplitudes $\mathbf{A}_{u}^{(0)}$ and $\mathbf{A}_{d}^{(N+1)}$. Resulting system of four linear equations is written in the matrix form ${ }^{18}$

$$
\left[\begin{array}{cc}
\mathbf{T}_{u d} \mathbf{S}_{d u}^{(1)}-\mathbf{I} & \mathbf{T}_{u u} \mathbf{S}_{u d}^{(2)} \\
\mathbf{T}_{d d} \mathbf{S}_{d u}^{(1)} & \mathbf{T}_{d u} \mathbf{S}_{u d}^{(2)}-\mathbf{I}
\end{array}\right]\left[\begin{array}{cc}
\mathbf{S}_{u u}^{(1)} & \mathbf{0} \\
\mathbf{0} & \mathbf{S}_{d d}^{(2)}
\end{array}\right]^{-1}\left[\begin{array}{c}
\mathbf{A}_{u}^{(0)} \\
\mathbf{A}_{d}^{(N+1)}
\end{array}\right]=\tilde{\mathbf{S}}_{T} \mathbf{A}_{o u t}=\mathbf{0} .
$$

For a nontrivial solution of this system of algebraic equations, which corresponds to laser resonance, the following condition for total matrix $\tilde{\mathbf{S}}_{T}$ must be fulfilled ${ }^{17,18}$

$$
\operatorname{det}\left\{\tilde{\mathbf{S}}_{T}\left(\lambda, g_{0}\right)\right\}=0
$$

Note that, in practice, it is equal to finding the resonant wavelength $\lambda$ and the threshold value of gain coefficient $g_{0}$, for which laser gain exactly compensates cavity losses. Once we find the resonant wavelength and the threshold value of the gain coefficient, Eq. (17) is used to determine the polarization state of the laser eigenmodes and the electric field distribution inside a cavity.

\subsection{Standing-Wave Pattern Calculation}

Procedure for extraction of laser eigenmodes of complicated anisotropic laser structures presented in Sec. 2.3 turns out to be very useful in determining several steady-state properties of spin-V(E)CSELs such as distribution of electromagnetic field inside laser cavity. Our method for the calculation of standing-wave pattern is described in Fig. 3. The important point is that the field in the entire laser structure can be obtained using emitted amplitudes. The procedure is following: i) we calculate laser eigenmode together with resonant wavelength and polarization state, ii) field is calculated for each $z$-coordinate using transfer or scattering matrices of each component of laser structure from both sides always up to the last QW (in the sense of considered direction of propagation). Note that in the active zone we obtain two sets of electromagnetic-field components originating in propagating field using transfer matrices from both sides of laser. For this reason iii) we average these two fields. It is necessary to perform the averaging because in the present approach each QW is approximated as an infinitesimally thin dipole layer placed at the center of QW film. Consequently, the field is not amplified smoothly but by steps, which causes certain inaccuracy. It is worth noting that the method is polarizationsensitive. Morover, one can apply recursive S-matrix algorithm ${ }^{18}$ to calculate exact field distribution in MQW system. 


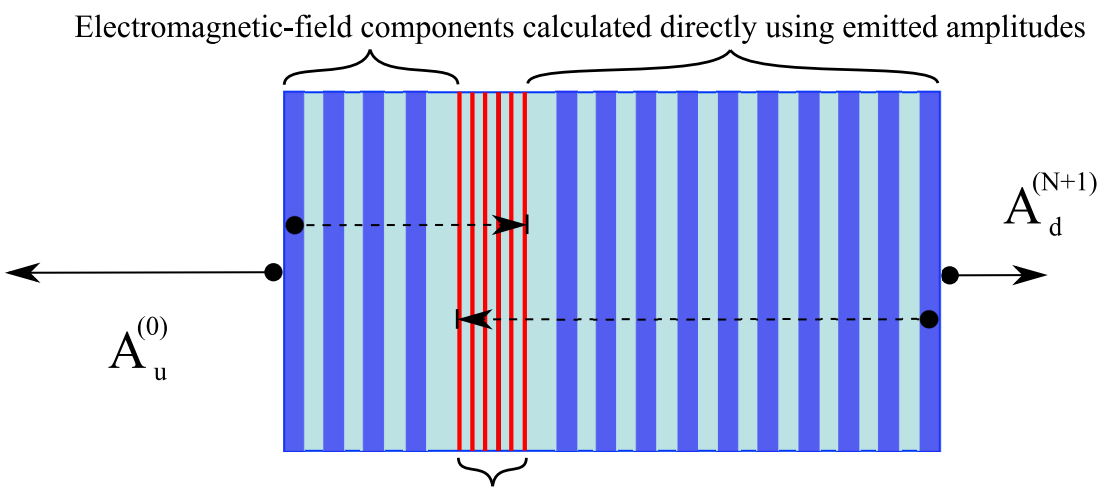

Averaged electromagnetic-field components

Figure 3. The method for calculation of electromagnetic-field distribution inside multi-QW spin-V(E)CSEL. Field components in Bragg mirrors or generally in passive laser components are calculated directly using emitted amplitudes. On the other hand, field inside multi-QW region is obtained as an average of fields given by both emitted amplitudes.

\subsection{Rate Equations of Two-Mode Spin-V(E)CSEL With Linear Gain Dichroism}

Let us now limit our considerations to dual-mode operation of spin-polarized V(E)CSEL with linear gain dichroism only and neglected birefringence. Such oscillation regime can be described in the limit of Class-A and Class-B lasers by Eqs. (2) and (5), where $i \in\{1,2\}$. However, it is impractical for example to solve time-dependence or stability of modes due to mathematical structure of the generalized Maxwell-Bloch equations, e.g. the presence of scalar product. For this reason we derive the rate equation model describing dynamics of anisotropic two-mode spin-polarized V(E)CSEL directly from Eqs. (2) and (5).

We introduce normalized intensities ${ }^{8} \tilde{I}_{(1,2)}=I_{(1,2)} / 2 I_{\text {sat }}$, where $I_{(1,2)}$ are the optical intensities of laser eigenmodes and $I_{s a t}$ is the saturation intensity. We define new population variables ${ }^{8} N=\frac{\tilde{g}}{2 \kappa}\left(N_{+}+N_{-}\right)$and $m=\frac{\tilde{g}}{2 \kappa}\left(N_{+}-N_{-}\right)$, where $\tilde{g}=2 \operatorname{Re}\{g\}$. It can be shown that we may consider $N, m$ 'eigenpopulations' of 4-level system in Fig. 1. We find the following system of equations ${ }^{24}$

$$
\begin{gathered}
\frac{d \tilde{I}_{(1)}}{d t}=\kappa\left[\left(\beta_{1}^{+}+\theta_{1}^{-}\right) N+\left(\beta_{1}^{+}-\theta_{1}^{-}\right) m\right] \tilde{I}_{(1)}-\kappa \tilde{I}_{(1)}, \\
\frac{d \tilde{I}_{(2)}}{d t}=\kappa\left[\left(\theta_{2}^{+}+\beta_{2}^{-}\right) N+\left(\theta_{2}^{+}-\beta_{2}^{-}\right) m\right] \tilde{I}_{(2)}-\kappa \tilde{I}_{(2)}, \\
\frac{d N}{d t}=\gamma N_{0}-\gamma\left[1+\left(\beta_{1}^{+}+\theta_{1}^{-}\right) \tilde{I}_{(1)}+\left(\theta_{2}^{+}+\beta_{2}^{-}\right) \tilde{I}_{(2)}\right] N-\gamma\left[\left(\beta_{1}^{+}-\theta_{1}^{-}\right) \tilde{I}_{(1)}+\left(\theta_{2}^{+}-\beta_{2}^{-}\right) \tilde{I}_{(2)}\right] m, \\
\frac{d m}{d t}=\gamma P_{\epsilon} N_{0}-\bar{\gamma}_{s} m-\gamma\left[\left(\beta_{1}^{+}+\theta_{1}^{-}\right) \tilde{I}_{(1)}+\left(\theta_{2}^{+}+\beta_{2}^{-}\right) \tilde{I}_{(2)}\right] m-\gamma\left[\left(\beta_{1}^{+}-\theta_{1}^{-}\right) \tilde{I}_{(1)}+\left(\theta_{2}^{+}-\beta_{2}^{-}\right) \tilde{I}_{(2)}\right] N,
\end{gathered}
$$

where $\beta_{1}^{+}, \beta_{2}^{-}$and $\theta_{1}^{-}, \theta_{2}^{+}$are the effective optical gain coefficients and the cross-coupling coefficients, ${ }^{18}$ respectively. Symbol $X_{i}^{ \pm}$denotes coefficient describing coupling between $i$-th mode and $N_{ \pm}$carrier population inversion, where $X \in\{\beta, \theta\}$. Note that eigenpolarizations can be obtained in the absence of any additional anisotropies in laser cavity such as the linear birefringence simply by finding eigenvectors of a gain tensor in Eq. (10). ${ }^{18}$ Overall pumping rate is quantified using $\gamma N_{0}$, where $N_{0}$ stands for the unsaturated population inversion. ${ }^{21}$ Spin polarization of pump is controlled by the parameter $P_{\epsilon}$ and so-called spin flip rate coefficient $\bar{\gamma}_{s}$ is defined as $\gamma+2 \gamma_{s}{ }^{8}$

Eqs. (19-22) describe coupling of two modes $\mathbf{E}_{(1,2)}$, generally differing in wavelength and polarization, to carrier population inversions $N_{ \pm}$. Such coupling between modes increases with increasing linear gain dichroism quantified using the parameter $\Delta .^{18}$ Note that in the absence of gain dichroism (and birefringence), which is equivalent to consider $\Delta=1$, there is no cross-coupling. It means that $\beta_{1}^{+}=\beta_{2}^{-}=1$ and $\theta_{1}^{-}=\theta_{2}^{+}=0$ and consequently (21), (22) become equivalent to (27), (28) (see Appendix B). One can see that also the rest of rate 
equations of our model become formally equivalent to spin flip model in the absence of linear gain anisotropy. In this sense, Eqs. (19-22) form the generalization to spin flip model where amplitude anisotropies are described by effective parameter $\gamma_{a}$ and are not localized. ${ }^{8,10}$ It should be noted that our approach to solve time-dependence of spin-V(E)CSELs can be adapted to include also linear birefringence that can be localized in arbitrary layer of laser structure. In the case of birefringent spin-V(E)CSEL structure, it is not possible to find eigenmodes as a gain tensor eigenvectors anymore and numerical methods must be used instead. It means that the calculation of effective gain coefficients and cross-saturation coefficients is much more complicated. However, this is out of the scope of this article.

\section{APPLICATION TO REAL SPIN-V(E)CSEL STRUCTURES}
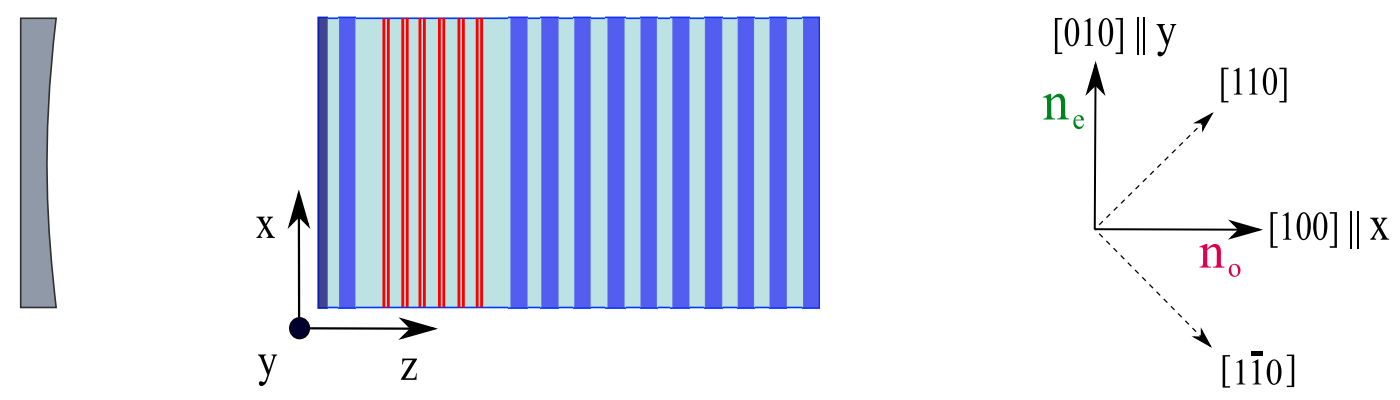

Figure 4. (left) A simplified structure of spin-VECSEL containing 12-QW active region with gain anisotropy used for application of proposed modeling tools. (right) Used reference frame. Source of linear birefringence is localized in two capping layers.

In the present part of this work we use modeling tools introduced in Sec. 2 to study both static and dynamical properties of real spin-V(E)CSELs. We limit ourselves to 1/2-VCSEL structures with external output coupler as shown schematically in Fig. 4. It contains active region with twelve InGaAs/GaAsP QWs and GaAs spacers. Optical feedback is realized by bottom Distributed Bragg Mirror(DBR) with 26 periods of GaAs/AlAs which provides almost perfect reflectivity. Reflectivity of output coupler placed typically few millimeters from $1 / 2$ VCSEL chip is $R=0.95$. Note that by changing distance between output coupler and $1 / 2$-VCSEL, one is able to modify photon lifetime $\tau_{p h}$ in optical cavity and consequently dynamical behavior of VECSEL. Entire structure is optimized to emit at $\lambda=980 \mathrm{~nm}$.

Our modeling tools respect anisotropic nature of V(E)CSEL structures. We consider both linear gain dichroism and linear birefringence in a calculation of electromagnetic-field distribution in laser structures and linear gain dichroism only in the context of dynamical properties of spin-VECSELs. Linear gain dichroism quantified using the parameter $\Delta$ is localized exactly inside each of QWs. On the other hand, linear birefringence quantified by the parameter $\delta n=n_{e}-n_{o}$ is localized inside two capping layers at the top of $1 / 2$-VCSEL. ${ }^{19}$ Total thickness of birefringent layers is $40 \mathrm{~nm}$. Note that according to Fig. 4, axes of ordinary and extraordinary refractive indices are not aligned along axes, in which linear gain dichroism was applied.

\subsection{Electromagnetic-Field Distribution Calculation}

Let us now apply method of electromagnetic-field distribution calculation described in Sec. 2.4 to abovementioned 12-QW VCSEL structure. Fig. 5 shows the resulting standing-wave pattern inside $1 / 2$-VCSEL for $\Delta=0.95, \delta n=0.025$ and the effective degree of spin polarization $P_{s}=0$. The polarization of the optical eigenmode is linear. Since axes of linear gain anisotropy and linear birefringence are not aligned, a vector of electric field intensity is oriented in quite general direction. We would obtain $E_{x}=E_{y}$ in each point of spinVECSEL, if we took $\delta n=0$. On the other hand, $E_{x}$ component increases with increasing linear birefringence parameter $\delta n$. We note that while searching for anisotropic spin-VECSEL eigenmodes, we usually find multiple sets of two transversal modes. Those sets are equidistant. We do not consider simultaneous oscillation of these two transversal modes in this subsection. Consequently, what we present is the field distribution of a single transversal mode. 


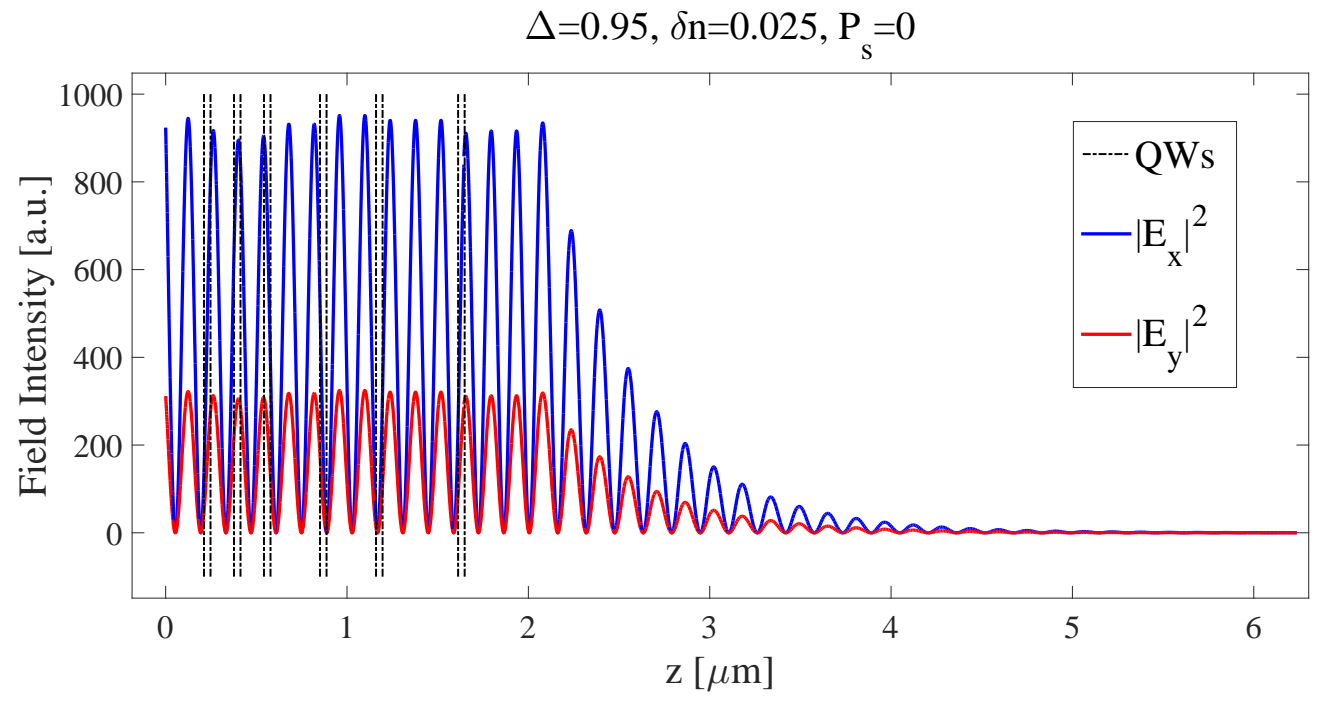

Figure 5. Electromagnetic-field distribution inside 1/2-VCSEL with linear gain dichroism and linear birefringence. Orthogonal directions are not equivalent due to optical anisotropies inside the laser cavity. We see that the wavelength of optical eigenmode lays inside photonic bandgap of DBR and thus electromagnetic field exponentially decays inside the Bragg mirror.

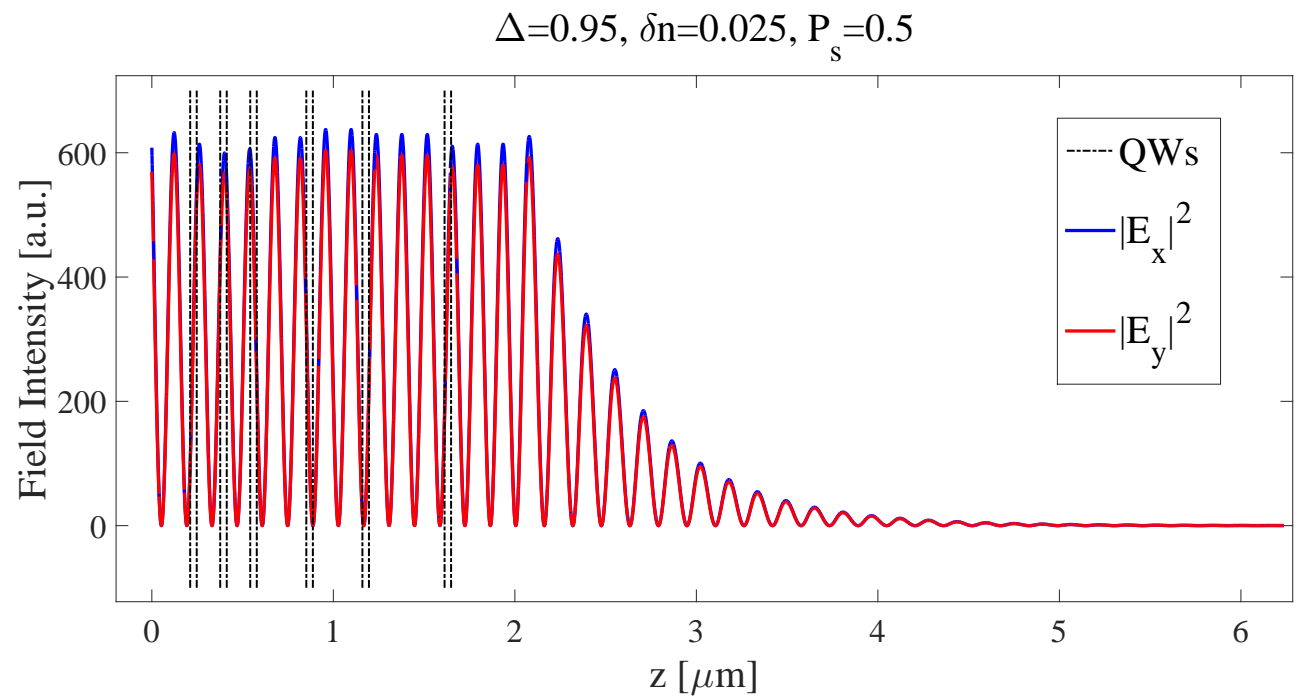

Figure 6. Electromagnetic-field distribution inside 1/2-VCSEL with linear gain dichroism and linear birefringence and pumped using spin-polarized current. Large effective spin polarization of conduction band electrons inside QWs is able to almost compensate the effects of linear optical anisotropies.

In the next case (see Fig. 6), we consider also non-zero effective spin polarization degree $P_{s}$. We see that using quite large value of $P_{s}$, we are able to almost compensate the effects of linear optical anisotropies. ${ }^{11,16}$ Polarization of emitted field is very close to perfectly circular one, which can be deduced from the values of the Stokes vector describing polarization state of emitted light.

\subsection{Dynamical Properties of Spin-V(E)CSELs}

This part of our contribution is dedicated to analysis of time-dependent phenomena in spin-V(E)CSELs. We study transient behavior of both laser eigenmodes under different conditions and polarization dynamics using time-dependent Stokes vectors components of emitted light. All the calculations are performed for spin-polarized 

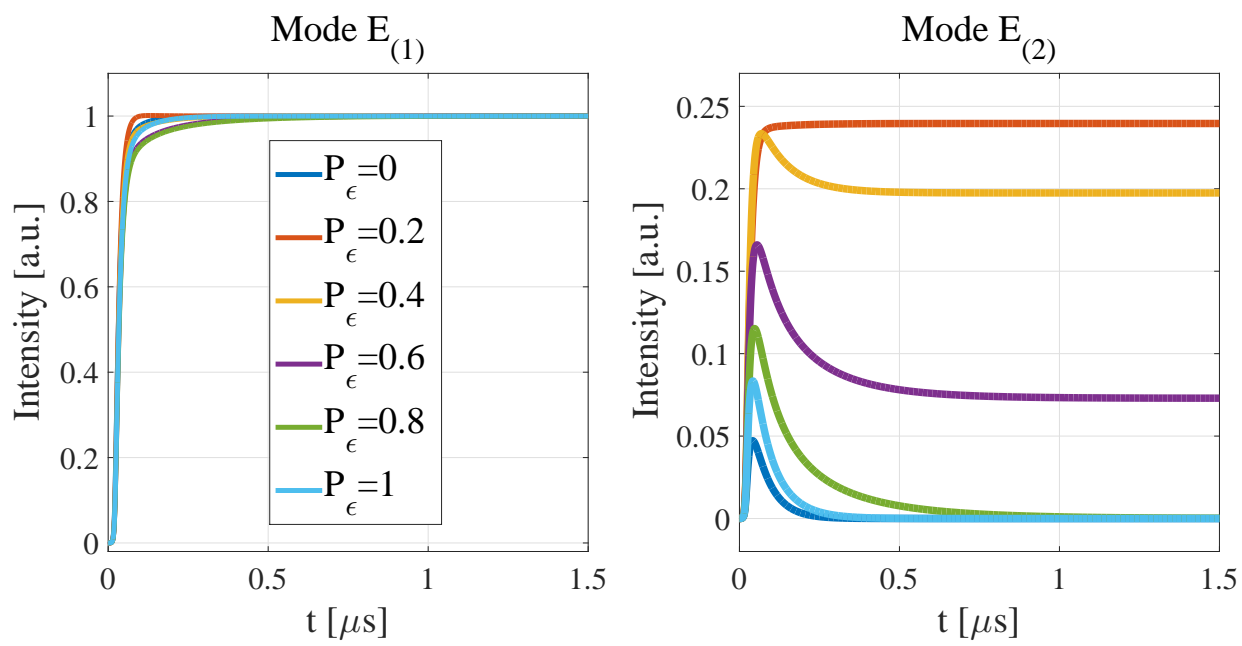

Figure 7. Time-dependence of two laser eigenmodes of spin-VECSEL calculated with fixed value of linear gain dichroism parameter $\Delta=0.9$. Pump polarization $P_{\epsilon}$ is variable parameter.

1/2-VCSEL with external cavity described in the previous subsection and shown in Fig. 4 (left). We emphasize here the effects of anisotropic properties of the laser cavity and active media such as the linear gain anisotropy and the spin polarization of conduction band electrons in QWs. Note that considering dynamical properties of spin-lasers linear birefringence is out of the scope of the present work.

The analysis is based on Eqs. (19-22) which are solve using conventional numerical Runge-Kutta methods. ${ }^{25}$ We use following values of parameters for simulations: ${ }^{6}$ the spin lifetime $\tau_{s}=1 / \bar{\gamma}_{s}=60 \mathrm{ps}$, the carrier lifetime $\tau=1 / \gamma=1 \mathrm{~ns}$ and the photon lifetime $\tau_{p h}=1 / \kappa=10 \mathrm{~ns}$. Note that we are able to calculate the electric field decay rate coefficient $\kappa$ or equivalently the photon lifetime $\tau_{p h}$ for arbitrary optical cavity using the scattering matrix formalism. It can be derived using Fourier analysis that the photon lifetime in a laser cavity is given by the cavity's transmission line shape. ${ }^{26}$ Normalized pumping parameter is fixed at value $N_{0}=5$, it means five times the threshold pumping rate. There are two controlled parameters of simulations, the pump polarization $P_{\epsilon}$ and the linear gain dichroism parameter $\Delta$.

In our first result (see Fig. 7), we consider realistic experimental situation of spin-polarized VECSEL with the gain dichroism characterized with $\Delta=0.9$. We solve time-dependence of both laser eigenmodes for several values of pump polarization $P_{\epsilon}$. In the first case, for $P_{\epsilon}=0$, eigenmode $\mathbf{E}_{(2)}$ quickly decays to zero. A possible physical interpretation is following: since there is zero spin polarization of electrons in QWs, two transversal linearly-polarized modes along [110] and [1ㅣㅣ] axes can oscillate in a cavity. ${ }^{18}$ This can be deduced from gain tensor defined in Eq. (10). These two possible modes interact with joint carrier reservoir described by carriers density $N=N_{+}+N_{-}$. However, they have different gain coefficient due to linear gain dichroism, which results in instability of mode with larger threshold population inversion. ${ }^{10}$ Such degeneracy of carrier reservoirs is lifted by non-zero spin pumping $\left(P_{\epsilon} \neq 0\right)$. As shown in Fig. 7, oscillation of both modes is possible for moderate values $\left(P_{\epsilon}=0.2, P_{\epsilon}=0.4, P_{\epsilon}=0.6\right)$ of pump polarization. In this sense, spin pumping is able to compensates the effects of linear gain anisotropy. On the other hand, if pump polarization is too large, only single mode oscillation regime occurs. The reason is that when we reduce the threshold pumping rate of preferential mode using spin pumping, we increase needed pumping rate for weaker mode. This effect is probably even stronger in presence of linear gain dichroism. ${ }^{6}$

Simulations presented in Fig. 8 consider experimentally rather impractical situation, because in this case linear gain dichroism is variable. It shows that fixed value of pump polarization of about $P_{\epsilon}=0.5$ is able to compensate (in the sense of two-mode oscillation regime) values of linear gain dichroism coefficient even slightly smaller that $\Delta=0.9$. We see that preferential mode $\mathbf{E}_{(1)}$ has typical Class-A laser characteristics which is given by values of the carrier and photon lifetimes. ${ }^{20,21}$ This is not the case for second mode which always oscillates 

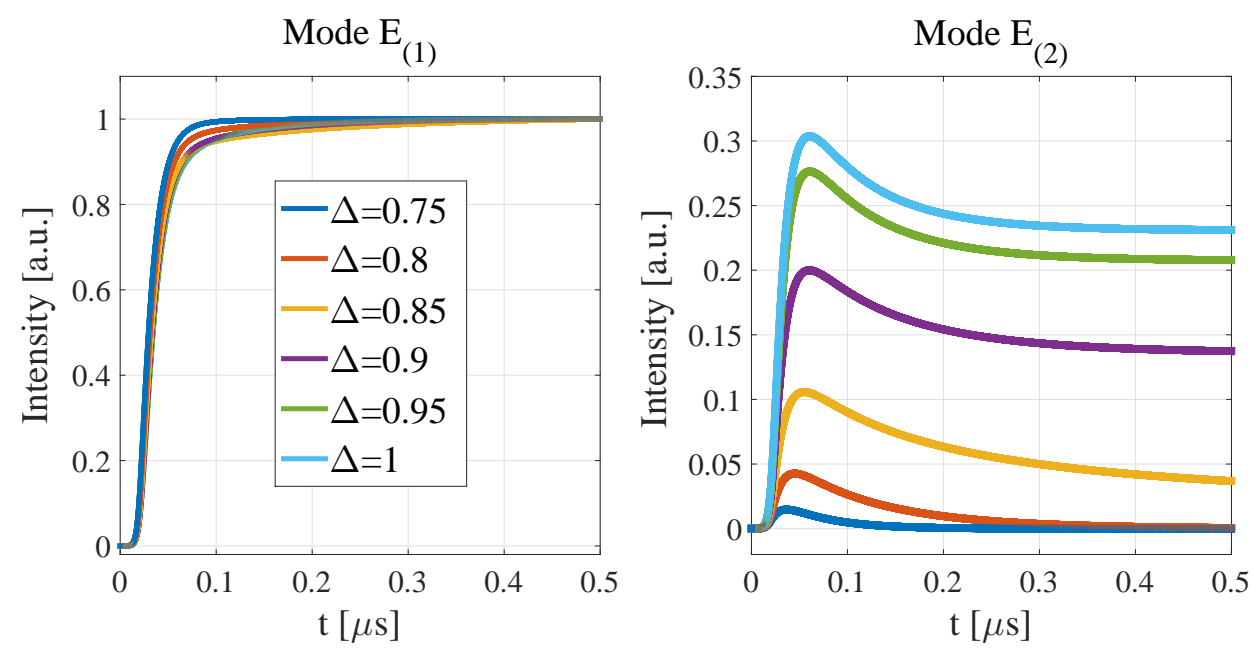

Figure 8. Time-dependence of two laser eigenmodes of spin-VECSEL calculated with fixed value of pump polarization $P_{\epsilon}=0.5$. Non-zero pump polarization together with linear gain dichroism result in preference of one mode $\mathbf{E}_{(1)}$ over the other one $\mathbf{E}_{(2)}$.

for a short time and then quickly decays to zero. It is caused by negative mode coupling - stronger mode makes the weaker one unstable.

In the following part we investigate polarization dynamics of spin-VECSELs. For this reason, we define the Jones vector of emitted field as

$$
\mathbf{J}=\sqrt{\frac{\tilde{I}_{(1)}}{\tilde{I}_{(1)}+\tilde{I}_{(2)}}} \mathbf{J}_{(1)}+\sqrt{\frac{\tilde{I}_{(2)}}{\tilde{I}_{(1)}+\tilde{I}_{(2)}}} \mathbf{J}_{(2)},
$$

where $\mathbf{J}_{(1)}$ and $\mathbf{J}_{(2)}$ are the Jones vectors of laser eigenmodes, which can be extracted directly from gain tensor in the absence of additional anisotropies in a laser cavity. Definition of $\mathbf{J}$ fulfills the normalization condition $\mathbf{J}^{*} \cdot \mathbf{J}=1$ in a case of orthogonal eigenpolarizations. Using Eq. (23), we are able to calculate components of the Stokes vector describing polarization of emitted laser field in terms of optical intensities.
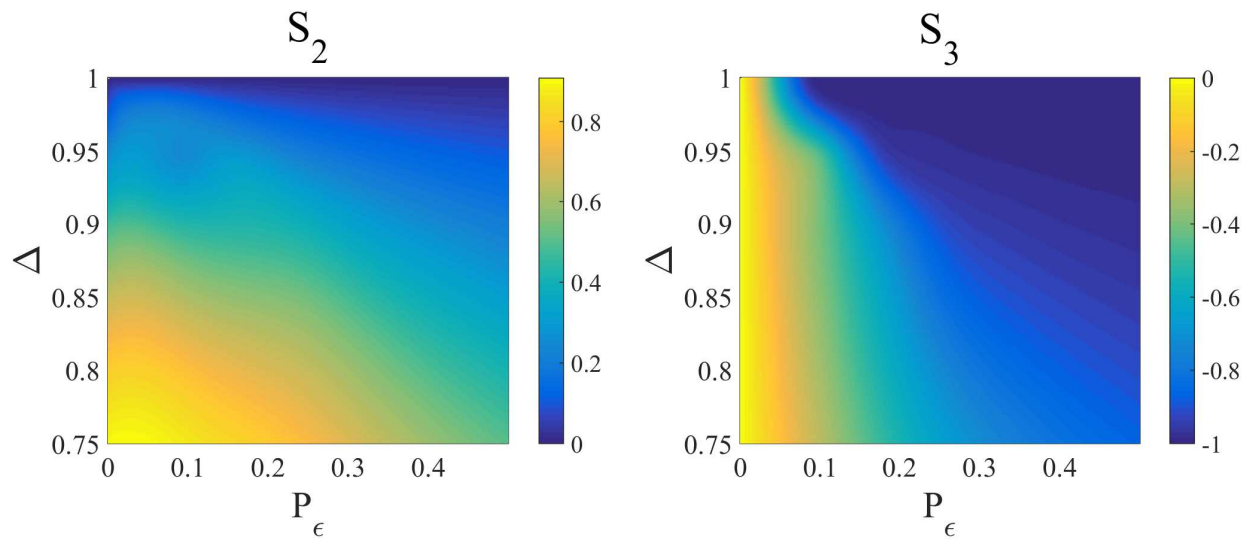

Figure 9. Stokes vector components $S_{2}, S_{3}$ expressed as functions of pump polarization $P_{\epsilon}$ and linear gain dichroism paramater $\Delta$. The result slightly differs from the calculations for each of eigenmodes separately. 

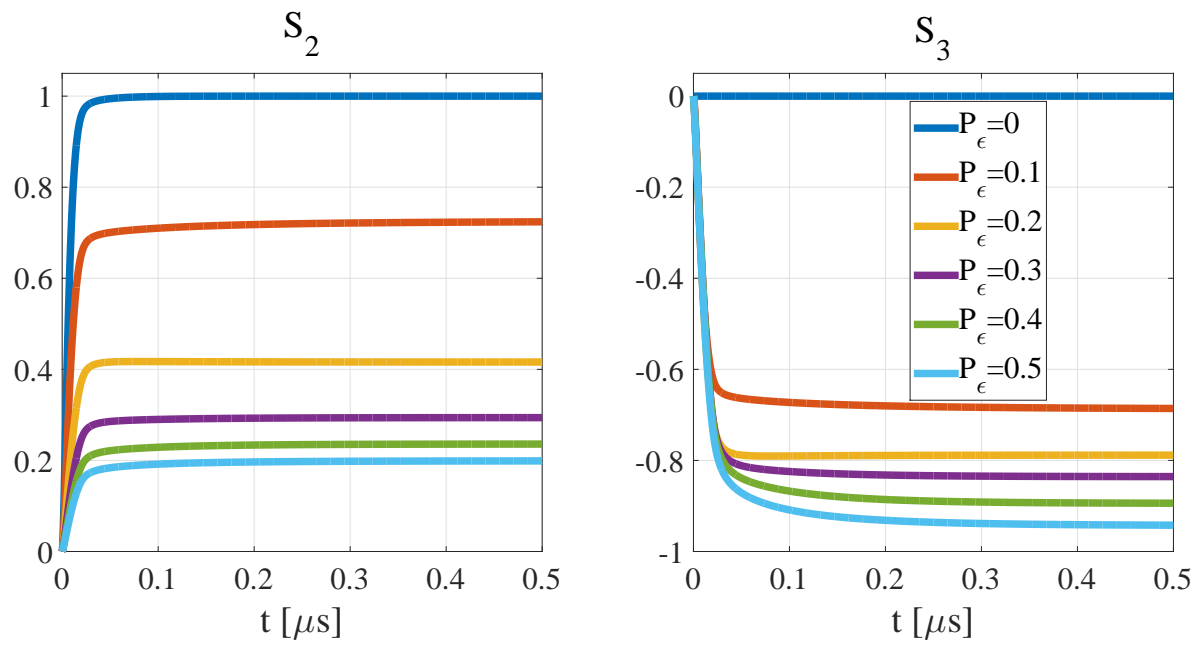

Figure 10. Time-dependence of Stokes vector components calculated for the fixed value of linear gain dichroism coefficient $\Delta=0.9$ and for several values of pump polarization $P_{\epsilon}$.

In order to determine polarization state of emitted field, we solve Eqs. (19-22) to obtain steady-state normalized intensities of laser eigenmodes. Next, we extract $\mathbf{J}_{(1)}$ and $\mathbf{J}_{(2)}$ using gain tensor. In Fig. 9, we show the calculated Stokes vector components $S_{2}=I_{x^{\prime}}-I_{y^{\prime}}, S_{3}=I_{+}-I_{-}$defined as intensity difference of linear modes along $x^{\prime}, y^{\prime}$ axes and difference of orthogonal circular polarizations, respectively. Note that axes $x^{\prime}, y^{\prime}$ are principal axes of linear gain anisotropy. Consequently, the Stokes vector element $S_{2}$ should be very sensitive to changes in $\Delta$. We see that this claim is confirmed by calculation. It means that $S_{2}$ changes more rapidly with changing $\Delta$ than with $P_{\epsilon}$. On the other hand, the element $S_{3}$ should be more sensitive to changes in spin pumping since circular polarizations are eigenpolarizations of spin-laser in the absence of any anisotropies. Again, it is verified by presented model. Note that obtained result depends on dynamical properties of spin-VECSELs such as mode coupling.

In Fig. 10, we present results concerning polarization dynamics. We express Stokes vector components as a function of time in interval until laser reaches its steady-state.

\section{CONCLUSIONS}

In this paper, we investigated dynamical and polarization properties of spin- $\mathrm{V}(\mathrm{E}) \mathrm{CSELs}$ using powerful scatteringmatrix approach which respects general semiclassical description of laser light amplification and dynamics. We used matrix methods to calculate electromagnetic field distribution inside real spin-VECSEL structure with localized phase and amplitude anisotropies, such as surface linear birefringence and linear gain dichroism in semiconductor QWs. Proposed method of standing-wave pattern calculation, which treats laser cavity as an active structure, can be used in the future to design and optimize future spin-lasers such as spin-V(E)CSELs. Also, we formulated generalized rate-equation model including linear gain dichroism in QWs of which the spinflip model is a special case. It will be modified in the near future to describe dynamical properties of spin-lasers with arbitrarily distributed anisotropies.

\section{APPENDIX A. SCATTERING MATRIX FORMALISM}

Let us consider general a anisotropic multilayer consisting of $\mathrm{N}$ layers interacting with planar electromagnetic waves. Optical phenomena such as interference, reflection and transmission can be described in the framework of the scattering matrix formalism. By solving Maxwell equations in each layer and using appropriate boundary conditions $^{2}$ one can construct the scattering matrix of a structure, which relates the incoming and the outgoing 


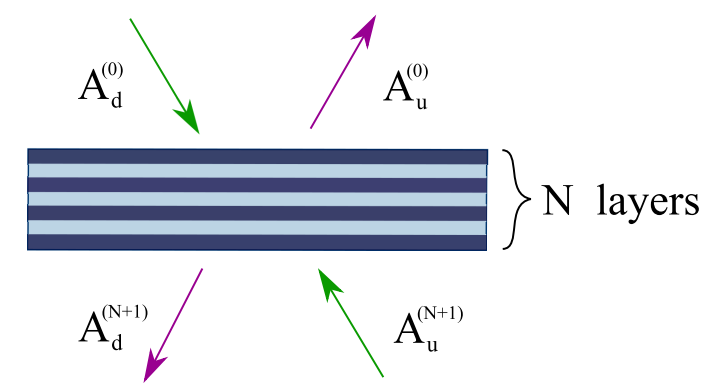

Figure 11. Scattering of electromagnetic field on generally anisotropic multilayer consisting of $\mathrm{N}$ thin layers. Incoming and outgoing amplitude components of the field are marked green and violet respectively and are related to each other using the scattering matrix.

amplitudes. The relation between the incoming and outgoing amplitudes as shown in Fig. 11 can be expressed as

$$
\left[\begin{array}{c}
\mathbf{A}_{u}^{(0)} \\
\mathbf{A}_{d}^{(N+1)}
\end{array}\right]=\left[\begin{array}{ll}
\mathbf{S}_{u u} & \mathbf{S}_{u d} \\
\mathbf{S}_{d u} & \mathbf{S}_{d d}
\end{array}\right]\left[\begin{array}{c}
\mathbf{A}_{u}^{(N+1)} \\
\mathbf{A}_{d}^{(0)}
\end{array}\right],
$$

where we use a notation with $2 \times 2$ submatrices and 2-component subvectors. General submatrix $\mathbf{S}_{\mu \nu}$ relates the incoming amplitude subvector $\mathbf{A}_{\nu}$ to outgoing subvector $\mathbf{A}_{\mu}$, where indices $\mu, \nu \in\{u, d\}$ define the direction of propagation - up or down.

\section{APPENDIX B. SPIN FLIP MODEL}

Originally developed in 1990s in order to describe polarization dynamics of conventional V(E)CSELs, modified version of spin flip model is frequently used to model dynamical properties of spin-polarized lasers. ${ }^{8,10}$ These 4-level rate equations couple the normalized amplitudes of orthogonal circularly-polarized fields $E_{+}, E_{-}$to two different population inversion variables $N \propto\left(N_{+}+N_{-}\right)$and $m \propto\left(N_{+}-N_{-}\right)$as described by Eqs. (25-28). The cavity decay rate is defined here as $\bar{\kappa}=\kappa / 2$ and the spin flip rate constant as $\bar{\gamma}_{s}=\gamma+2 \gamma_{s}$.

$$
\begin{gathered}
\frac{d E_{+}}{d t}=\bar{\kappa}(1+i \alpha)(N+m) E_{+}-\bar{\kappa} E_{+}-\left(\gamma_{a}+i \gamma_{p}\right) E_{-}, \\
\frac{d E_{-}}{d t}=\bar{\kappa}(1+i \alpha)(N-m) E_{-}-\bar{\kappa} E_{-}-\left(\gamma_{a}+i \gamma_{p}\right) E_{+}, \\
\frac{d N}{d t}=\gamma N_{0}-\gamma\left\{\left[1+\left|E_{+}\right|^{2}+\left|E_{-}\right|^{2}\right] N+\left[\left|E_{+}\right|^{2}-\left|E_{-}\right|^{2}\right] m\right\}, \\
\frac{d m}{d t}=\gamma P_{\varepsilon} N_{0}-\bar{\gamma}_{s} m-\gamma\left\{\left[\left|E_{+}\right|^{2}+\left|E_{-}\right|^{2}\right] m+\left[\left|E_{+}\right|^{2}-\left|E_{-}\right|^{2}\right] N\right\} .
\end{gathered}
$$

In contrary to our approach, spin flip model treats amplitude and phase anisotropies using effective parameters $\gamma_{a}$ and $\gamma_{p}$ which must be often fitted to experimental results.

\section{ACKNOWLEDGMENTS}

We acknowledge the support from the projects IT4Innovations National Supercomputing Center-Path to Exascale Project No. CZ.02.1.01/0.0/0.0/16 013/0001791, ESF in "Science without borders" project, reg. nr. CZ.02.2.69/0.0/0.0/16 027/0008463 within the Operational Programme Research, Development and Education, Student Project No. SP2018/51, and Czech Science Foundation Grant No. 18-22102S. 


\section{REFERENCES}

[1] Michalzik, R., [VCSELs: Fundamentals, Technology and Applications of Vertical-Cavity Surface-Emitting Lasers], Springer-Verlag Berlin Heidelberg (2013).

[2] Yeh, P., [Optical Waves in Layered Media], John Wiley and Sons (1988).

[3] Holub, M. and Bhattacharya, P., "Spin-polarized light-emitting diodes and lasers," Journal of Physics D: Applied Physics 40(11), R179 (2007).

[4] Gerhardt, N. C. and Hofmann, M. R., "Spin-controlled vertical-cavity surface-emitting lasers," Advances in Optical Technologies 2012 (2012).

[5] Holub, M., Shin, J., Chakrabarti, S., and Bhattacharya, P., "Electrically injected spin-polarized verticalcavity surface-emitting lasers," Applied Physics Letters 87(9), 091108 (2005).

[6] Frougier, J., Baili, G., Alouini, M., Sagnes, I., Jaffrès, H., Garnache, A., Deranlot, C., Dolfi, D., and George, J.-M., "Control of light polarization using optically spin-injected vertical external cavity surface emitting lasers," Applied Physics Letters 103(25), 252402 (2013).

[7] Meier, F. and Zakharchenya, B., [Optical Orientation], North Holland (1984).

[8] San Miguel, M., Feng, Q., and Moloney, J. V., "Light-polarization dynamics in surface-emitting semiconductor lasers," Phys. Rev. A 52, 1728-1739 (Aug 1995).

[9] Travagnin, M., van Exter, M. P., Jansen van Doorn, A. K., and Woerdman, J. P., "Role of optical anisotropies in the polarization properties of surface-emitting semiconductor lasers," Phys. Rev. A 54, 1647-1660 (Aug 1996).

[10] Martin-Regalado, J., Prati, F., Miguel, M. S., and Abraham, N. B., "Polarization properties of verticalcavity surface-emitting lasers," IEEE Journal of Quantum Electronics 33, 765-783 (May 1997).

[11] Joly, A., Baili, G., Alouini, M., George, J.-M., Sagnes, I., Pillet, G., and Dolfi, D., "Compensation of the residual linear anisotropy of phase in a vertical-external-cavity-surface-emitting laser for spin injection," Opt. Lett. 42, 651-654 (Feb 2017).

[12] Iba, S., Koh, S., Ikeda, K., and Kawaguchi, H., "Room temperature circularly polarized lasing in an optically spin injected vertical-cavity surface-emitting laser with (110) gaas quantum wells," Applied Physics Letters 98(8), 081113 (2011).

[13] Gerhardt, N. C., Li, M. Y., Jähme, H., Höpfner, H., Ackemann, T., and Hofmann, M. R., "Ultrafast spininduced polarization oscillations with tunable lifetime in vertical-cavity surface-emitting lasers," Applied Physics Letters 99(15), 151107 (2011).

[14] Yokota, N., Nisaka, K., Yasaka, H., and Ikeda, K., "Spin polarization modulation for high-speed verticalcavity surface-emitting lasers," Applied Physics Letters 113(17), 171102 (2018).

[15] Lindemann, M., Xu, G., Pusch, T., Michalzik, R., Hofmann, M. R., Žutić, I., and Gerhardt, N. C., "Ultrafast spin-lasers," https://arxiv.org/abs/1807.02820 (pre-print) (2018).

[16] Alouini, M., Frougier, J., Joly, A., Baili, G., Dolfi, D., and George, J.-M., "VSPIN: a new model relying on the vectorial description of the laser field for predicting the polarization dynamics of spin-injected V(E)CSELs," Opt. Express 26, 6739-6757 (Mar 2018).

[17] Fördös, T., Postava, K., Jaffrès, H., and Pištora, J., "Matrix approach for modeling of emission from multilayer spin-polarized light-emitting diodes and lasers," Journal of Optics 16(6), 065008 (2014).

[18] Fördös, T., Jaffrès, H., Postava, K., Seghilani, M. S., Garnache, A., Pištora, J., and Drouhin, H.-J., "Eigenmodes of spin vertical-cavity surface-emitting lasers with local linear birefringence and gain dichroism," Phys. Rev. A 96, 043828 (Oct 2017).

[19] Fördös, T., Postava, K., Jaffrès, H., Quang To, D., Pištora, J., and Drouhin, H. J., "Mueller matrix ellipsometric study of multilayer spin-vcsel structures with local optical anisotropy," Applied Physics Letters 112(22), 221106 (2018).

[20] Sargent, M., Scully, M. O., and Lamb, W. E., [Laser physics], London : Addison-Wesley (1974).

[21] Siegman, A., [Lasers], University Science Books (1986).

[22] Yu, J.-L., Chen, Y.-H., Tang, C.-G., Jiang, C., and Ye, X.-L., "Observation of strong anisotropic forbidden transitions in (001) ingaas/gaas single-quantum well by reflectance-difference spectroscopy and its behavior under uniaxial strain," Nanoscale Research Letters 6, 210 (Mar 2011). 
[23] Henry, C., "Theory of the linewidth of semiconductor lasers," IEEE Journal of Quantum Electronics 18, 259-264 (February 1982).

[24] Drong, M., Fördös, T., Jaffrès, H., Postava, K., Peřina Jr., J., Pištora, J., and Drouhin, H.-J., "Stability, dynamics and spatial distribution of optical eigenmodes in spin-polarized V(E)CSELs involving local linear anisotropies," (in preparation) (2019).

[25] Butcher, J., [Numerical Methods for Ordinary Differential Equations], John Wiley \& Sons (2008).

[26] Saleh, B. E. A. and Teich, M. C., [Fundamentals of photonics; 2nd ed.], Wiley series in pure and applied optics, Wiley, New York, NY (2007). 